\title{
Improving the efficiency of the cardiac catheterization laboratories through understanding the stochastic behavior of the scheduled procedures
}

\author{
Pieter S. Stepaniak ${ }^{1,2}$, Mohamed A. Soliman Hamad ${ }^{3}$, \\ Lukas R.C. Dekker ${ }^{4}$, Jacques J. Koolen ${ }^{4}$ \\ ${ }^{1}$ Department of Operating Rooms, Catharina Hospital, Eindhoven, The Netherlands \\ ${ }^{2}$ Medical University of Gdansk, Poland \\ ${ }^{3}$ Department of Cardio-thoracic Surgery, Catharina Hospital, Eindhoven, The Netherlands \\ ${ }^{4}$ Department of Cardiology, Catharina Hospital, Eindhoven, The Netherlands
}

\begin{abstract}
Background: In this study, we sought to analyze the stochastic behavior of Catherization Laboratories (Cath Labs) procedures in our institution. Statistical models may help to improve estimated case durations to support management in the cost-effective use of expensive surgical resources.

Methods: We retrospectively analyzed all the procedures performed in the Cath Labs in 2012. The duration of procedures is strictly positive (larger than zero) and has mostly a large minimum duration. Because of the strictly positive character of the Cath Lab procedures, a fit of a lognormal model may be desirable. Having a minimum duration requires an estimate of the threshold (shift) parameter of the lognormal model. Therefore, the 3-parameter lognormal model is interesting. To avoid heterogeneous groups of observations, we tested every group-cardiologist-procedure combination for the normal, 2- and 3-parameter lognormal distribution.

Results: The total number of elective and emergency procedures performed was 6,393 $(8,186 \mathrm{~h})$. The final analysis included 6,135 procedures $(7,779 \mathrm{~h}$ ). Electrophysiology (intervention) procedures fit the 3-parameter lognormal model 86.1\% (80.1\%). Using Friedman test statistics, we conclude that the 3-parameter lognormal model is superior to the 2-parameter lognormal model. Furthermore, the 2-parameter lognormal is superior to the normal model.

Conclusions: Cath Lab procedures are well-modelled by lognormal models. This information helps to improve and to refine Cath Lab schedules and hence their efficient use. (Cardiol J 2014; 21, 4: 343-349)
\end{abstract}

Key words: catheterization laboratory, scheduling, lognormal, efficiency

\section{Introduction}

Catheterization Laboratories (Cath Labs) are relative capital and labor-intensive departments within the hospital. Therefore, these departments are financially important for the hospital. Due to the increasing prevalence of cardiovascular diseases (CVD) as well as rapidly expanding absolute numbers and types of catheter procedures, the demand for catheterization procedures is increasing [1].

Address for correspondence: Pieter S. Stepaniak, PhD, Department of Operating Rooms, Catharina Hospital, PO BOX 1350, 5602 ZA Eindhoven, The Netherlands, e-mail: stepaniak@casema.nl 
By 2030, 40.5\% of the US population is expected to have some form of CVD [1]. With regard to this information, it may be expected that the demand for relatively scarce costly Cath Labs in the future will also increase. For this reason, Cath labs should be used optimally. This means that the utilization should be maximized, whereas idle times (i.e., under-utilized time or high turnover times) and work outside regular hours (i.e., over-utilized time) should be minimized. It has been shown that frequent work beyond scheduled hours does not only lead to overtime costs, but also to intangible costs resulting from dissatisfaction and reduced motivation of staff [2]. Overtime work is one of the primary reasons for nurses to terminate their employment [2] and scheduling conflicts are a major cause of nursing staff turnover [3]. Therefore, management of Cath Labs should aim at maximal use of available Cath Labs time given the aforementioned constrains.

The production in our Cath Lab has increased in the last years without a corresponding increase in its capacity. To help more patients within the same capacity, several process redesigns aiming to improve efficiency have been applied. In the literature, various studies have reported different methods used to improve the efficiency in the Cath Labs [4-9]. To the best of our knowledge, the stochastic behavior of Cath Labs procedures has not been described yet. Understanding this stochastic behavior is a fundamental starting point for optimizing Cath Labs schedules. Procedures performed in the Cath Labs are stochastic, as compared to procedures in the operating room (OR), which have been statistically modelled by many studies [10-15]. Similar to the OR, efficient use of Cath Labs crucially depends on estimated case durations. Statistical models may help to improve these estimates to support management in the cost-effective use of expensive surgical resources [15]. In this study, we sought to analyze the stochastic behavior of Cath Labs procedures in our institution. The concept of this study is based on an earlier study modelling the procedure and surgical times in the operating room [14-15].

\section{Methods}

We retrospectively analyzed all the procedures performed in 2012 in the Cath Labs of Catharina Hospital in Eindhoven, the Netherlands. The procedure time was an important factor in our analysis. We defined procedure time as the time lapse between the moment of entry of the patient to the
Cath Lab room and departing it after the treatment [16]. In our hospital, there are 5 Cath Lab rooms. The daily Cath Lab capacity is assigned to a certain cardiologist. Patients are scheduled on a firstcome-first-served basis unless there are reasons to do it differently; e.g., acute cases, availability of cardiologists or patient. The scheduled time for a procedure-cardiologist combination is based on the average duration of the last ten similar procedures performed by the same cardiologist.

Within the Cath Labs, there were two different groups of treatment: electrophysiology and percutaneous intervention (PCI). The procedure duration of Cath Lab procedures is strictly positive (larger than zero) and has mostly a large minimum duration. Because of the strictly positive character of these procedures, a fit of a lognormal model may be desirable. Having a minimum duration requires an estimate of the threshold (shift) parameter of the lognormal model. Therefore, the 3-parameter lognormal model is of interest.

The general formula for the lognormal model can be described as follows:

$$
\begin{aligned}
& f x(\mathrm{x} ; \mu, \delta, \theta)=\frac{1}{(\mathrm{x}-\theta) \delta \sqrt{2 \pi}} e^{-\frac{(\ln (x-\theta)-\mu)^{2}}{2 \delta^{2}}} \\
& (\mathrm{x}>\theta)
\end{aligned}
$$

where $\theta=$ shift parameter for duration data $\theta>0$. The case where $\theta$ equals zero is called the 2 -parameter lognormal model. For the 3-parameter lognormal model, we estimated the shift parameter by using a modified version of the approach of Spangler [13]. The shift parameter describing the location or origin of the random variable is important for the decisionmaking because it provides a lower bound on values of the random variable [13]. Firstly, we calculated the natural logarithm of procedure time for every cardiologist-procedure combination. Then we used the bisection method to estimate the shift parameter. In this way, we estimated three parameters for each combination of cardiologist and procedure.

\section{The bisection method}

- Set Lower $=0$.

- Set Upper is the smallest observed value.

- Initial Guess $=($ LOWER+UPPER $) / 2$.

- Subtract GUESS from all observed values, take the logarithm, and estimate the mean and standard deviations.

- Recalculate the Shapiro Wilk p-value $\left(=\mathrm{p}_{\text {new }}\right)$.

- Repeat this iteratively using bisection to find the shift parameter that results in the largest value of the $p$-value. 
- Stop the iteration if $\left(p_{\text {new }}-p_{\text {old }}\right) / p_{\text {new }} \times 100 \%$ $<1 \%$ or if $p_{\text {new }}<p_{\text {old }}$. If the final p-value is larger than 0.05 , we do not reject the hypothesis of the normal model.

To avoid heterogeneous groups of observations, we tested every group-cardiologist procedure combination for the normal, 2- and 3-parameter lognormal distribution. Very small sample sizes $(n<10)$ were excluded because they may indiscriminately fit almost every model. Normality tests were performed by using the method of Shapiro-Wilk. We defined the null hypothesis of the Shapiro-Wilk test statistic (W) as a sample from a normally distributed population. Thus, $\mathrm{p}>0.05$ for $\mathrm{W}$ accepts this assumption of normality. Most studies agree that this is the most reliable method to test the normality of small to medium-sized samples [17-20]. To detect the influence of sample size on the Shapiro--Wilk test, we arbitrarily divided the sample size into small $(10 \leq \mathrm{n}<30)$, medium $(30 \leq n<200)$, and large $(n \geq 200)$. To perform the analyses, we used StatsDirect statistical software and IBM SPSS 20. Normal probability plots were examined visually for procedures that were not well-fitted by either the normal or lognormal models. We analyzed QQ-PP and Box plots to confirm the results of the Shapiro--Wilk test. Examination of the calculated skewness and kurtosis, and of the histogram, box plot, and normal probability plot for the data may provide clues as to why the data failed the Shapiro-Wilk. Time points were exact to the minute.

Using Friedman test, we compared the (log) normal models to determine if there is a superior model. The null-hypothesis is that the models are equal. Here, the relevant question is whether the 2-parameter lognormal model is superior to the normal model and whether the 3-parameter lognormal model is superior to the 2-parameter lognormal model. A p value $<0.05$ was considered significant.

\section{Results}

The total number of elective and emergency procedures was $6,393(8,186 \mathrm{~h})$. Eight procedures were excluded from the analysis because the end time was not registered. This resulted in 6,385 cases $(8,175 \mathrm{~h})$. Procedures with frequency smaller than 10 were also excluded ( 250 cases; 396 h). The final analysis included 6,135 procedures $(7,779 \mathrm{~h})$. To have a homogenous group of observations per procedure, we created cardiologist-procedure combinations, which were differentiated into elective and acute cases.

The number of acute cases was $1,410(1,058 \mathrm{~h})$ and the number of elective cases was $4,725(6,721 \mathrm{~h})$. Seventeen cardiologists were involved in all the procedures. The number of cardiologist-procedure combinations was 303 (152 electrophysiology, and 151 invasive). The performed procedures are classified as follows (Tables $1-3$ ):

\section{Elective}

- Electrophysiology $(\mathrm{n}=3,192$ and time $=2,908 \mathrm{~h}$ ): 36 types of procedures, performed by 7 ope-rators.

- Intervention $(\mathrm{n}=1,533$ and time $=3,814 \mathrm{~h})$ : 17 type of procedures, performed by 10 operators.

Table 1. Procedure-cardiologist combinations, electrophysiology.

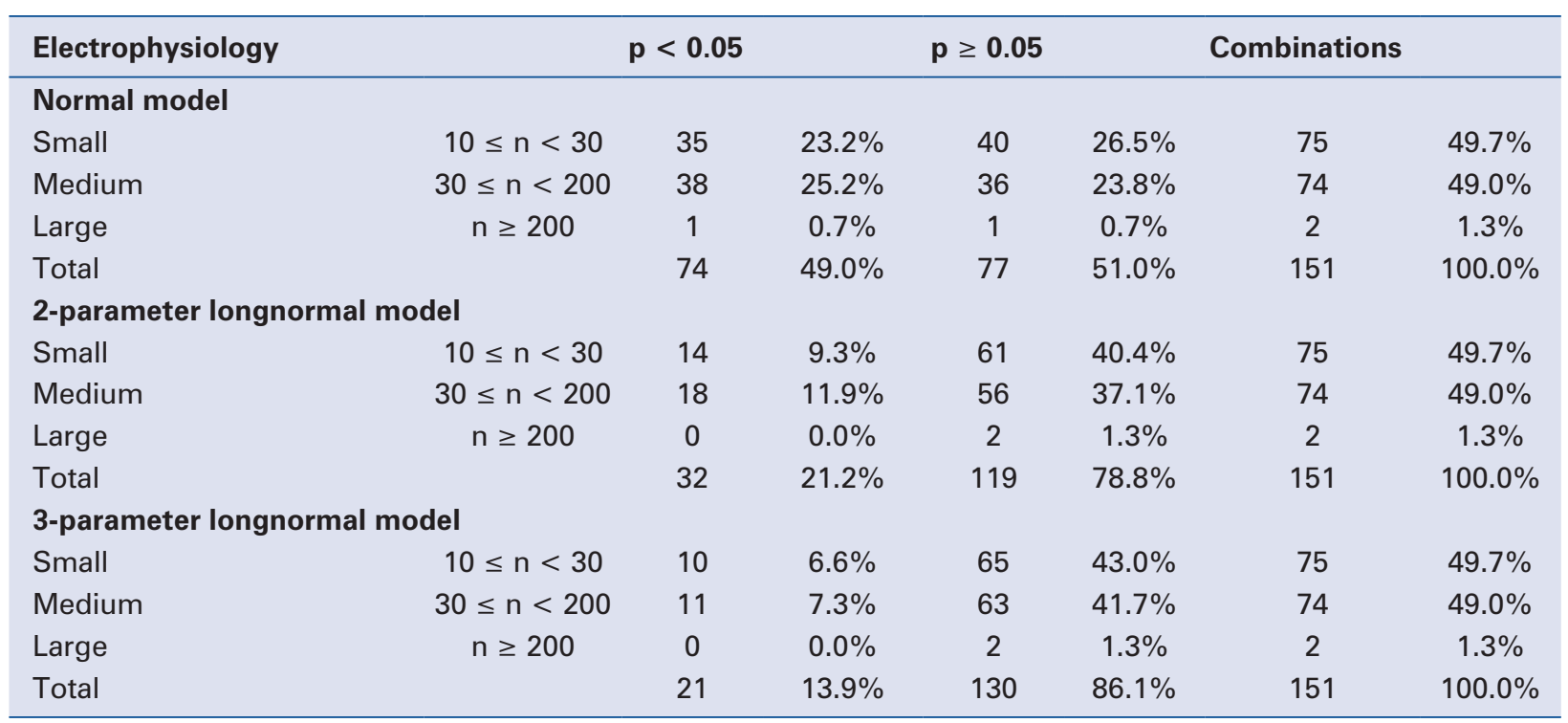


Table 2. Procedure-cardiologist combinations, interventions.

\begin{tabular}{|c|c|c|c|c|c|c|c|}
\hline \multicolumn{2}{|l|}{ Intervention } & \multicolumn{2}{|c|}{$p<0.05$} & \multicolumn{2}{|l|}{$p \geq 0.05$} & \multicolumn{2}{|c|}{ Combinations } \\
\hline \multicolumn{8}{|c|}{ Normal model } \\
\hline Small & $10 \leq n<30$ & 24 & $15.9 \%$ & 42 & $27.8 \%$ & 66 & $43.7 \%$ \\
\hline Medium & $30 \leq \mathrm{n}<200$ & 45 & $29.8 \%$ & 40 & $26.5 \%$ & 85 & $56.3 \%$ \\
\hline Large & $n \geq 200$ & 0 & $0.0 \%$ & 0 & $0.0 \%$ & 0 & $0.0 \%$ \\
\hline Total & & 69 & $45.7 \%$ & 82 & $54.3 \%$ & 151 & $100.0 \%$ \\
\hline \multicolumn{8}{|c|}{ 2-parameter longnormal model } \\
\hline Small & $10 \leq \mathrm{n}<30$ & 10 & $6.6 \%$ & 56 & $37.1 \%$ & 66 & $43.7 \%$ \\
\hline Medium & $30 \leq \mathrm{n}<200$ & 32 & $21.2 \%$ & 53 & $35.1 \%$ & 85 & $56.3 \%$ \\
\hline Large & $n \geq 200$ & 0 & $0.0 \%$ & 0 & $0.0 \%$ & 0 & $0.0 \%$ \\
\hline Total & & 42 & $27.8 \%$ & 109 & $72.2 \%$ & 151 & $100.0 \%$ \\
\hline \multicolumn{8}{|c|}{ 3-parameter longnormal model } \\
\hline Small & $10 \leq \mathrm{n}<30$ & 7 & $4.6 \%$ & 59 & $39.1 \%$ & 66 & $43.7 \%$ \\
\hline Medium & $30 \leq \mathrm{n}<200$ & 23 & $15.2 \%$ & 62 & $41.1 \%$ & 85 & $56.3 \%$ \\
\hline Large & $n \geq 200$ & 0 & $0.0 \%$ & 0 & $0.0 \%$ & 0 & $0.0 \%$ \\
\hline Total & & 30 & $19.9 \%$ & 121 & $80.1 \%$ & 151 & $100.0 \%$ \\
\hline
\end{tabular}

Table 3. Acute cases.

\begin{tabular}{lcccccc}
\hline Intervention (only medium size) & & $\mathbf{p}<0.05$ & $\mathbf{p} \geq \mathbf{0 . 0 5}$ & & $\begin{array}{c}\text { Combina- } \\
\text { tions }\end{array}$ \\
\hline Normal model & $30 \leq \mathrm{n}<200$ & 16 & $88.9 \%$ & 2 & $11.1 \%$ & 18 \\
2-parameter longnormal model & $30 \leq \mathrm{n}<200$ & 7 & $38.9 \%$ & 11 & $61.1 \%$ & 18 \\
3-parameter longnormal model & $30 \leq \mathrm{n}<200$ & 4 & $2.6 \%$ & 14 & $77.8 \%$ & 18 \\
\hline
\end{tabular}

\section{Acute intervention}

Eighteen cardiologist-procedure combinations, 10 operators, 1,410 procedures, $1,058 \mathrm{~h}$.

Figures 1-5 show examples of significant procedure-cardiologist combinations. Figure 6 shows a QQ plot of the lognormal procedure time of an acute PCI.

Electrophysiology (intervention) procedures fit the normal model $51.0 \%$ (54.3\%), 2-parameter lognormal model $78.8 \%$ (72.2\%) and the 3-parameter lognormal model $86.1 \%$ (80.1\%). For acute cases, we have only a medium-size group of observations. When analyzing the acute cases, we found that the 3-parameter lognormal distribution fits in $77 \%$ of the cases.

Using Friedman test statistics, we compared the following hypotheses:

- 3-parameter lognormal model equals the normal model $(\mathrm{p}=0.0001)$;

- 3-parameter lognormal model equals the 2 -parameter lognormal model $(\mathrm{p}=0.0054)$;

- 2-parameter lognormal model equals the normal model $(\mathrm{p}=0.0042)$.

This means that that the 3 -parameter lognormal model is superior to the 2-parameter lognormal model. The 2-parameter lognormal is superior to the normal model.

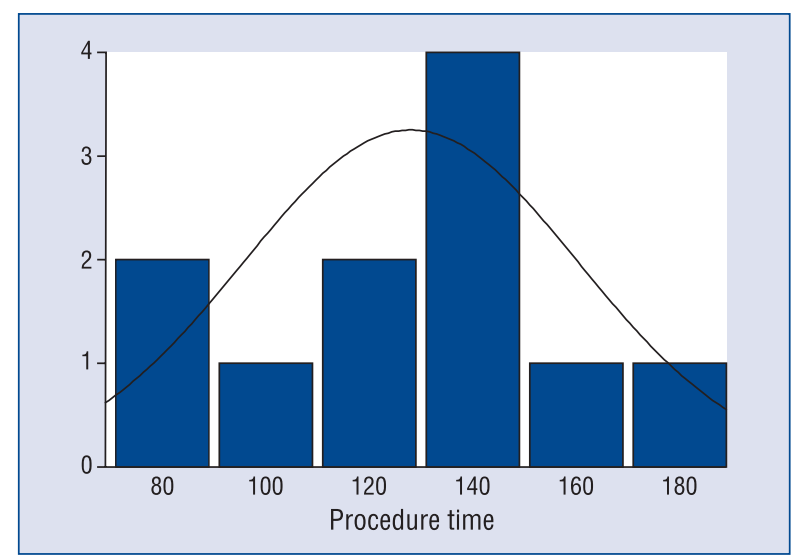

Figure 1. Ablation slow pathway, cardiologist $A ; W=$ $=0.932117, \mathrm{p}=0.4327$.

\section{Discussion}

The main finding of this study was that procedure times in de Cath Labs are mostly lognormaldistributed where the 3-parameter lognormal distribution is superior to the 2-parameter lognormal and the normal distribution. Understanding the 


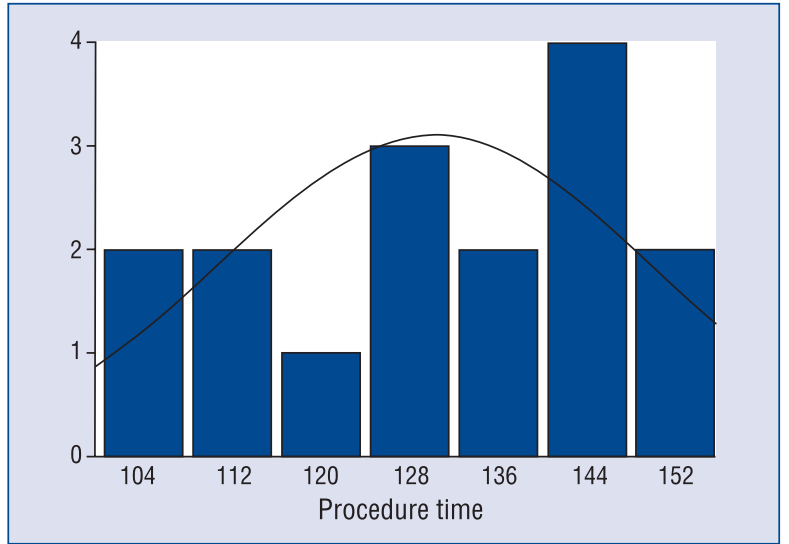

Figure 2. Ablation pulmonary vein isolation met pulmonary vein ablation catheter, cardiologist $B$.

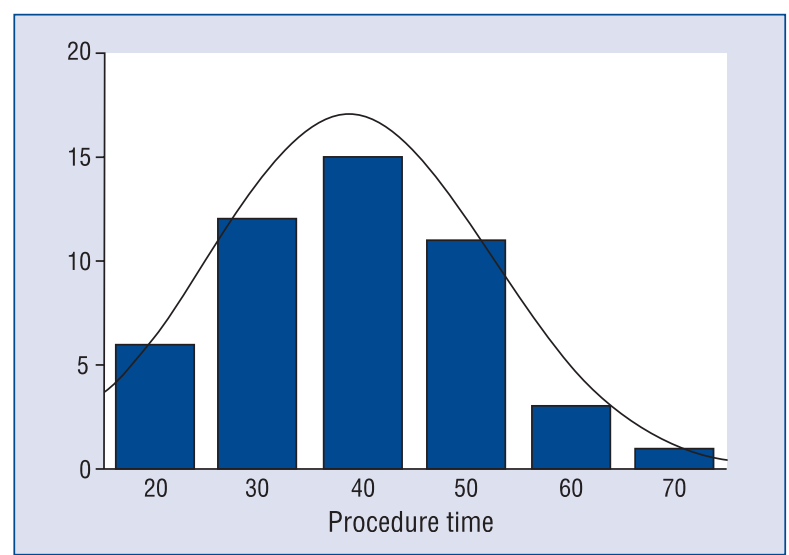

Figure 3. Coronary angiography, cardiologist C.

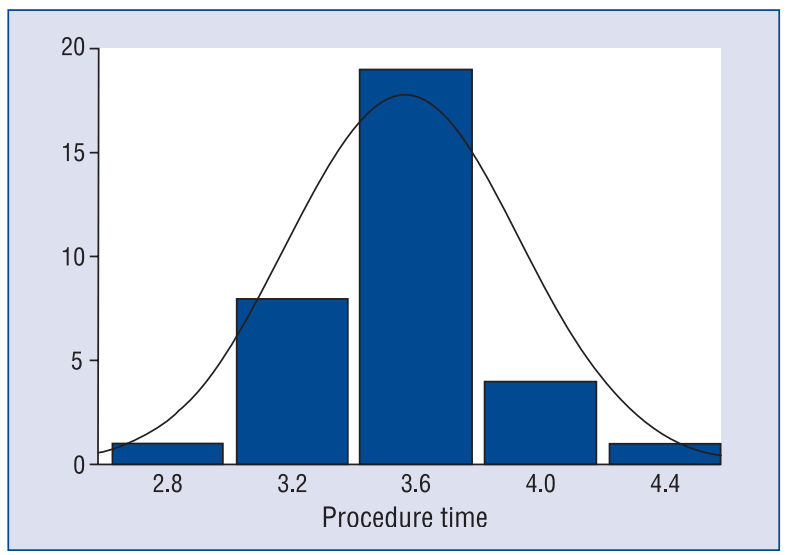

Figure 4. Coronary angiography, cardiologist E.

stochastic behavior of Cath Labs procedures is of utmost importance because of many reasons. We hereby discuss the conceptual and operational

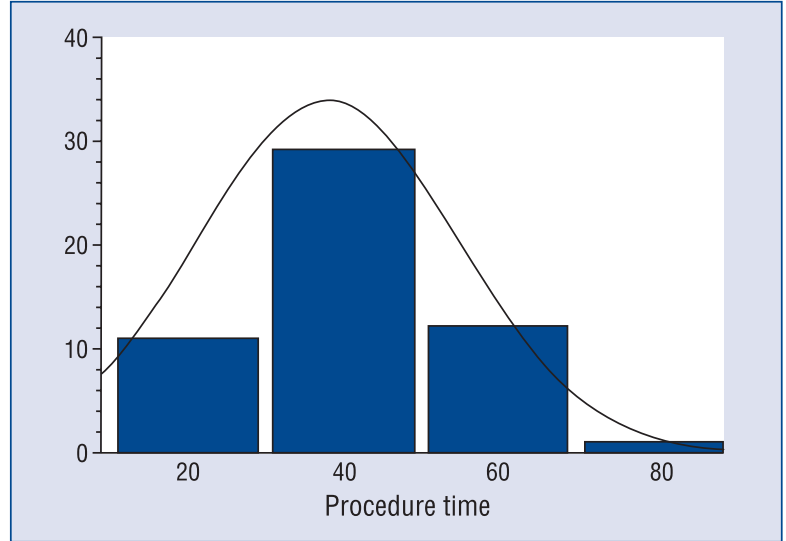

Figure 5. Acute percutaneous coronary intervention, cardiologist D.

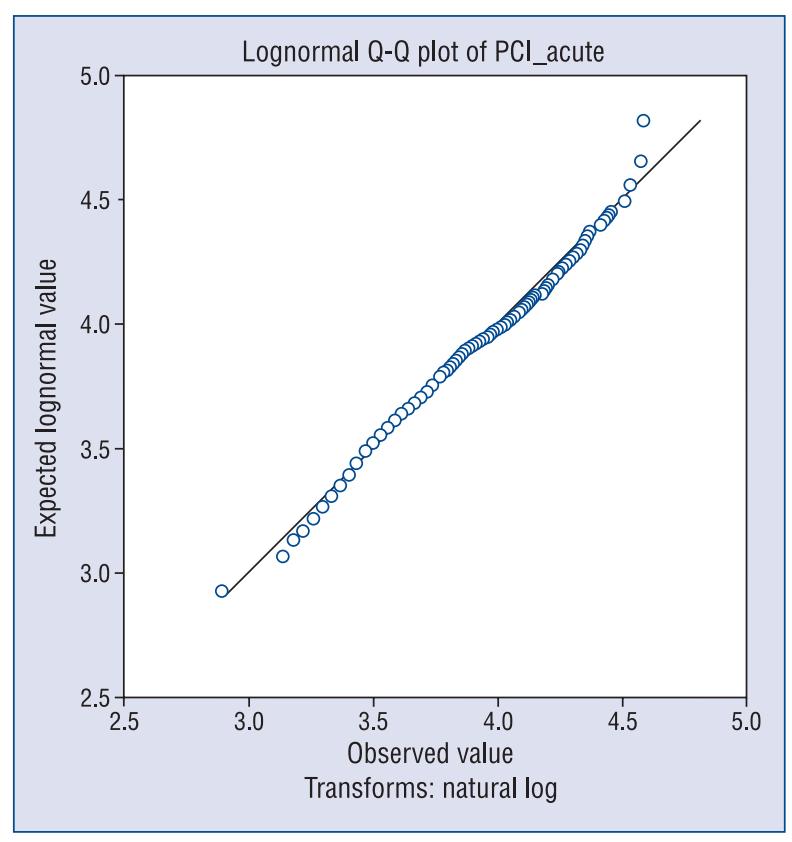

Figure 6. Lognormal Q-Q plot of acute percutaneous coronary intervention (PCl) of cardiologist $\mathrm{D}$.

reasons. From the conceptual point of view, we refer to the findings of The Institute of Medicine (IoM) report "Crossing the Quality Chasm: A New Health System for the $21^{\text {st }}$ Century". In this report, many problems in the quality of the United States' health care delivery system are described [21]. This report suggested that health care should be safe, effective, patient-centered, timely, efficient and equitable. In the present study, we focused on the timely, efficient en patient-centered dimensions addressed by the IoM. According to the IoM, timely access to care is reducing waits and possible harm- 
ful delays for both those who receive and those who deliver the care. One of the most serious problems has to do with timely access to hospital services. Problems involving access to care manifest in a variety of forms, including rejection of patients. Because not all the patients are equal, variation in treatment duration exists and managing this variation by using statistical models increases efficient care.

From an operational point of view, a good statistical model for procedure times makes procedure times more predictable by making a more accurate estimation of the produce duration. Next, it helps manage the variation of Cath Labs procedures. The aforementioned reasons contribute to reliable Cath Lab schedules. Reliable schedules allow the efficient use of scarce Cath Lab capacity because it reduces the chance of overused Cath Lab time and the chance that cases are cancelled. Reducing overtime is important because overtime is relative costly. Second, if cases are not well-scheduled, there will be a chance that scheduled patients will be cancelled and will stay longer in the hospital. Prolonged hospital stay does not only lead to elevated costs and hence less revenue, but also to less satisfaction of patients. As Cath Labs are scarce and hence have limited capacities, the information of this study may be used to achieve schedules that are more efficient. Examples of these methods are scheduling the same cases consecutively in fasttrack pathways [22-24], scheduling cases in specific rooms [25], Stochastic Integer Programming [26]. In the Cath Lab under study, there is no holding were patients are prepared for the treatment. This means that procedure times may be longer than in the situation with a preparation room. As a result, the threshold for the 3-lognormal model in our study may be larger. For future research, it could be interesting to analyze if procedure duration depends on several cardiologist-related factors such as work rate experience. In other words, it is important to know whether there is a relation between the number of procedures a cardiologist performed and the procedure duration or typical patient-related factors. This knowledge will help to improve further the estimation of Cath $\mathrm{Lab}$ procedure durations and hence manage variation in healthcare processes.

\section{Limitations of the study}

This is a retrospective single-center study. In our hospital, the usual case mix of procedures within a Cath Lab is performed as compared to other Cath Labs. Whether a similar analysis in other institutions would confirm our findings is to be investigated.

\section{Practical use in a clinical setting}

The findings in the paper help management and cardiologists to make better Cath Lab schedules. When patients are scheduled then management want to use the assigned capacity maximally without a large chance that overtime is needed or elective patients are cancelled. As shown in this paper, Cath Lab procedures have a skewed distribution: mean values are relatively low, variances are relatively large and values are strictly positive. Because of the log-normal characteristic of procedure times, once more time is needed for a coronary angiography, then it can be hard to 'win' time on the next scheduled coronary angiography to be ready on time after a days' work.

Nowadays, we make elective schedules and take into account the underlying distribution of a cardiologist-procedure combination. In sequencing cases, we avoid, for example, 2 procedures, which are highly skewed. Applying the knowledge from this paper in our department resulted in less overtime and a reduction in the number of cancelled patients. Teams experienced less stress to finish the schedule on time as well. Further research in simulating optimal Cath Lab schedules will help to improve further the Cath Lab efficiency.

\section{Conclusions}

Cath Lab procedures are well-modelled by lognormal models. This information helps to improve and to refine Cath Lab schedules and hence their efficient use.

\section{Conflict of interest: none declared}

\section{References}

1. Heidenreich PA, Trogdon JG, Khavjou OA et al. Forecasting the future of cardiovascular disease in the United States. A policy statement from the American Heart Association. Circulation, 2011; 123: 933-944.

2. Stachota P, Normandin P. Reasons registered nurses leave or change employment status J Nurs Admin, 2003; 33: 111-118.

3. Thomson TP, Brown H. Turnover of licensed nurses in skilled nursing facilities. Nurs Econ, 2002; 20: 66-69.

4. Siegel B, Wilson JM, Sickler. Enhancing work flow to reduce crowding. Jt Comm J Qual Patient Saf, 2007; 33 (suppl. 1): 57-67.

5. LeBlanc F, McLauglin S, Freedman J, Sager R, Weissman M. A six sigma approach to maximizing productivity in the cardiac cath lab. Cardiovasc Manag, 2004; 15: 19-24. 
6. Newell A. Cardiac catheterization laboratory management: The fundamentals. Radiol Manage, 2012; 34: 38-43.

7. Bradley EH, Nallamothu BK, Herrin J et al. National efforts to improve door-to-balloon time results from the door-to-balloon alliance. J Am Coll Cardiol, 2009; 54: 2423-2429.

8. Rossetti RR, Hill B, Johansson A, Dunkin R, Ingalls G eds. Utilization of discrete event simulation in de prospective determination of optimal cardiovascular lab processes. Proceedings of the 2009. Winter Simulation Conference, 2009: 1916-1926.

9. Lapierre SD, Batson C, McCaskey S. Improving on-time performance in health care organisations: A case study. Health Care Manage Sci, 1999; 2: 27-34.

10. Strum DP, Sampson AR, May JH, Vargas LG. Surgeon and type of anesthesia predict variability in surgical procedure times. Anesthesiology, 2000; 92: 1454-1466.

11. Strum DP, May JH, Sampson AR, Vargas LG, Spangler WE. Estimating times of surgeries with two component procedures: Comparison of the lognormal and normal models. Anesthesiology, 2003; 98: 232-240.

12. May JH, Strum DP, Vargas LG. Fitting the lognormal distribution to surgical procedure times. Decision Sci, 2000; 31: 129-148.

13. Spangler WE, Strum DP, Vargas LG, May JH. Estimating procedure times for surgeries by determining location parameters for the lognormal model. Health Care Manage Sci, 2004; 7: $97-104$.

14. Strum DP, May JH, Vargas LG. Modeling the uncertainty of surgical procedure times: comparison of lognormal and normal models. Anesthesiology, 2000; 92: 1160-1167.

15. Stepaniak PS, Heij C, Mannaerts GHH, de Quelerij M, de Vries G. Modeling procedure and surgical times for CPT-anesthesiasurgeon combinations and evaluation in terms of case duration prediction and OR efficiency: A multi-center study. Anesth Analg, 2009; 109: 1232-1245.
16. Donham RT, Mazzei WJ, Jones RL. Procedural times glossary. Am J Anesthesiol, 1999; 23 (5 suppl.): 4-12.

17. D'Agostino RB. Tests for the normal distribution. In: D'Agostino RB, Stephens MA eds. Goodness-of fit techniques. Marcel Dekker, Inc., New York 1986: 367-419.

18. Shapiro SS, Wilk MB. An analysis of variance test for normality. BioMetrika, 1965; 52: 591-599.

19. Royston JP, Shapiro-Wilk. Normality test and P-value. Applied Statistics, 1995; 4: 44.

20. Shapiro SS, Wilk MB, Chen HJ. A comparative study of various tests for normality. J Am Stat Assoc, 1968; 63: 1343-1372.

21. Committee on Quality of Health Care in America, Institute of Medicine. Crossing the Quality Chasm: A New Health System for the 21st Century. National Academies Press, Washington, DC, USA 2001.

22. Stepaniak PS, Heij C, Buise MP, Mannaerts GHH, Smulders JF, Nienhuijs SW Bariatric surgery with operating room teams that stayed fixed during the day: A multicenter study analyzing the effects on patient outcomes, teamwork and safety climate, and procedure duration. Anesth Analg, 2012; 115: 1384-1392 .

23. Stepaniak PS, Vrijland W, de Quelerij M, de Vries G, Heij C. Working with a fixed operating room team on consecutive similar cases and the effect on case duration and turnover time Arch Surg, 2010; 145: 1165-1170.

24. Haanschoten MC, van Straten AH, ter Woorst JF et al. Fast-track practice in cardiac surgery: Results and predictors of outcome. Interact Cardiovasc Thorac Surg, 2012; 15: 989-994.

25. Dexter F, Traub RD. How to schedule elective surgical cases into specific operating rooms to maximize the efficiency of use of operating room time. Anesth Analg, 2002; 94: 933-942.

26. Schultz R. Stochastic programming with integer variables. Math Program, 2003; 97: 285-309. 